Gwizdka, J. (1998). Categorization Is Difficult: Use of an Electronic Notebook For Organizing Design Meeting Notes. Proceedings of Human Factors and Ergonomics Society 42th Annual Meeting, Chicago, October 5-10 1998. Santa Monica, CA: HFES. 516-520.

\title{
CATEGORIZATION IS DIFFICULT: USE OF AN ELECTRONIC NOTEBOOK FOR ORGANIZING DESIGN MEETING NOTES
}

\author{
Jacek Gwizdka \\ Department of Mechanical and Industrial Engineering \\ University of Toronto \\ 5 Kings College Rd, Toronto, ON M5S 3G8, CANADA \\ jacek@ie.utoronto.ca
}

\begin{abstract}
The electronic engineering notebook is a pen-based computer tool designed to capture engineering notes and to assist in structuring them to facilitate subsequent information access. Experiments were conducted in which free-form and fixed-form note-taking interfaces were compared with respect to capturing and structuring notes from a design meeting. Suitability of domain-based and user-defined terminologies for semantic structuring was also tested. Results from the experiment confirmed that a free-form interface was easier to use for note-taking and structuring notes than were forms with fixed structure. However, difficulties with applying semantic categorizations were observed in both types of interface. The results indicated that terminology should be chosen appropriately to a task, and that experienced users have less problems with applying it. The experiment also demonstrated high diversity in note-taking styles, thus highlighting the need to accommodate individual preferences in electronic notebooks.
\end{abstract}

\section{INTRODUCTION}

In spite of its importance, engineering design information is often difficult to retrieve. This is especially true of information recorded in paper engineering notebooks that do not support the structuring needed to facilitate access to notes.

Recent progress in computing technologies has enabled development of portable computer tablets with pen-input. This research explored how electronic notebooks employing these new technologies could assist engineers in capturing and retrieving structured notes from design meetings.

\section{RELATED WORK}

A number of researchers have studied electronic notebook use in the context of personal (Erickson, 1996 and Wilcox et al., 1996) and design (Lakin et al., 1992 and Hong et al., 1995) note taking. In Dynomite (Wilcox et al., 1996) properties describing type of information could be assigned to handwritten notes and user-defined keywords to pages. The system had a set of pre-defined general purpose properties, other could be added by users. Proteus (Erickson, 1996) used "stamps" to mark notes. The author noted that cognitive overhead associated with applying stamps prevented them from being used in practice. Personal Electronic Notebook with Sharing (Hong et al., 1995) supported note taking, browsing, and sharing notes through the Internet. Structuring notes was performed by assigning user defined categories. Although keywords, or other structuring elements, were employed in these electronic notebooks, empirical studies on keyword terminology usage and on its effects on information retrieval strategies were generally lacking.

In a previous work on Electronic Engineering Notebooks (EEN) performed in our research laboratory, Louie (1995) compared paper and electronic notebook with regard to reading, writing, and sketching activities. In reading and sketching both media were found to be equally good; in writing, paper was a better medium. Louie focused on design information acquisition, while research reported in this paper concentrated on structuring of design information.

Terminology used in electronic notebooks should be appropriate for the task, in order to facilitate subsequent retrieval. In an earlier study, researchers manually indexed documents from a design project with domain model terms and demonstrated resulting improvement in information retrieval (Baudin et al., 1993). In the study reported below, the domain model was used by engineers to index their own notes in an EEN. The goal was to assess the fitness of terminology as a means of indexing design meeting notes. 


\section{SYSTEM}

An electronic notebook for engineers was designed and implemented with three variants of user interface. The first was a free-form interface that did not impose any constraints on user input (Figure 1). It allowed for attaching labels to handwritten objects. The label terminology based on design ontologies (Lin, Fox, and Bilgic, 1996; Gwizdka and Dalal, 1996) allowed for description of the following entities: 1) concepts in the design process (requirement, rationale, issue), 2) product structure (part, parameter), and 3) project management items (action, meeting). Each concept was further characterized by attributes and by relations to other concepts. Users could attach attribute labels to objects and link objects to represent their relationships. The second interface was similar, but with user defined terms for labels.

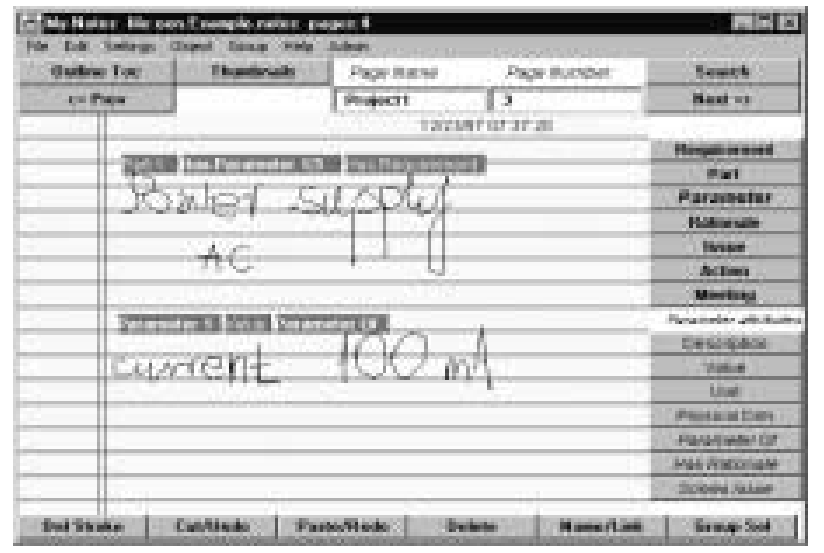

Figure 1. EEN Free-form User Interface

The third interface was fixed-form (Figure 2), using the same domain terminology as in the first. Each of the main concepts had a corresponding form with attributes and relations represented as fields. In each interface variant, notes were organized into pages that could be named by users. EEN was implemented in Java, running on a computer with pen input.

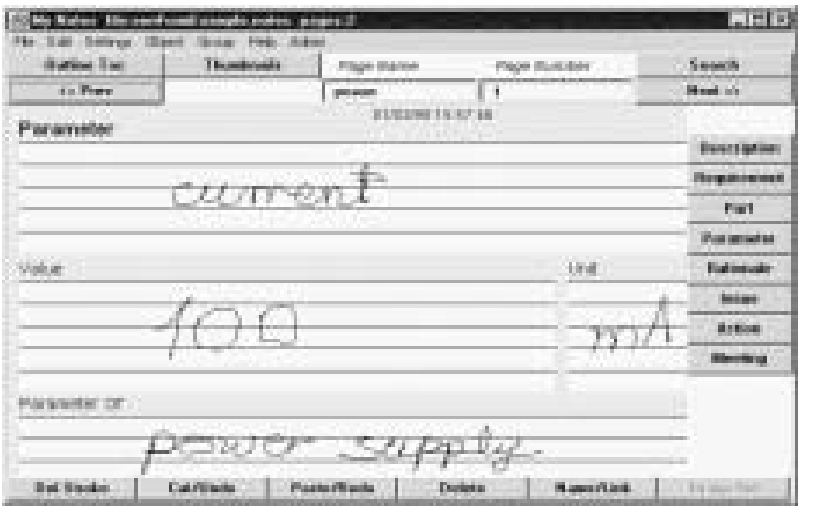

Figure 2. EEN Fixed-form User Interface
METHOD

A design meeting note-taking study was conducted with 20 participants (undergraduate and graduate students in the Department of Mechanical and Industrial Engineering, at the University of Toronto). The study consisted of two one hour sessions for each participant (with the second session being held for each participant several days after the first). Participants were chosen on the basis of their engineering design experience. The minimum experience required was a university engineering design course.

The study had three conditions, each defined by one of the variants of the EEN interface.

1. Free-form interface with pre-defined, domain-based semantic labels (FFP).

2. Free-from interface with user defined labels (FFU). 3. Fixed-form interface with domain-based forms and fields, based on the same terminology as in the first condition (FORM)

Seven students participated in the first and the third, and six in the second, conditions (Table 1). Participants were randomly allocated across the three interface conditions.

\begin{tabular}{|l|l|l|l|}
\hline Interface Condition & Novice & Expert & Total \\
\hline FFP & 4 & 3 & $\mathbf{7}$ \\
FFU & 2 & 4 & $\mathbf{6}$ \\
FORM & 4 & 3 & $\mathbf{7}$ \\
Total & $\mathbf{1 0}$ & $\mathbf{1 0}$ & $\mathbf{2 0}$ \\
\hline
\end{tabular}

Table 1. Allocation of participants across interface conditions

In the first session, after 15 minutes of training on the usage of domain terminology, on the task, and on the interface itself, participants performed a note-taking task while watching a videotape of a short (10 min.) design meeting. The objective of the videotaped meeting was to discuss a design of a programmable thermostat interface for a house heating system. Discussed topics included: components of the artifact, their parameters and values, requirements and rationale for components and parameters, actions required and meetings scheduled. Participants were allowed to pause and rewind the tape. After note-taking, participants in the free-form conditions organized their notes by attaching labels, while participants in the form condition went through their notes and counted the types of forms. In the second session participants performed information retrieval from their notes. 


\section{RESULTS}

The following data was collected in the experiment: notes taken by participants during the first session, notes made by the experimenter on observations of participants and during debriefing in both sessions, participant answers to interview questions, and audio tapes from the second session. Experimenter notes from the second session included a record of the steps taken by participants in retrieving the information needed to answer questions.

The results were analyzed with respect to two independent factors: user interface condition (FFP, FFU, and FORM) and participants' engineering experience. We defined expert participants as students who had additional engineering design experience gained either by work in industry or while performing university research beyond the standard design courses.

A range of dependent variables measuring different aspects of note-taking, note-structuring and information retrieval from notes was defined. The following were the main metrics used in evaluation:

1. NCOV - Number of design meeting concepts covered in notes. It indicated degree of completeness of the notes.

2. CATSD - Depth of search using structuring elements (labels, form types or fields). It was calculated as the number of different categories (terms) used during a search.

3. TRUSTR - Number of categorization-based retrieval strategies (also called "trust" strategies) used. "Trust" strategies are information finding strategies that employ categorizations, for example, search for labels.

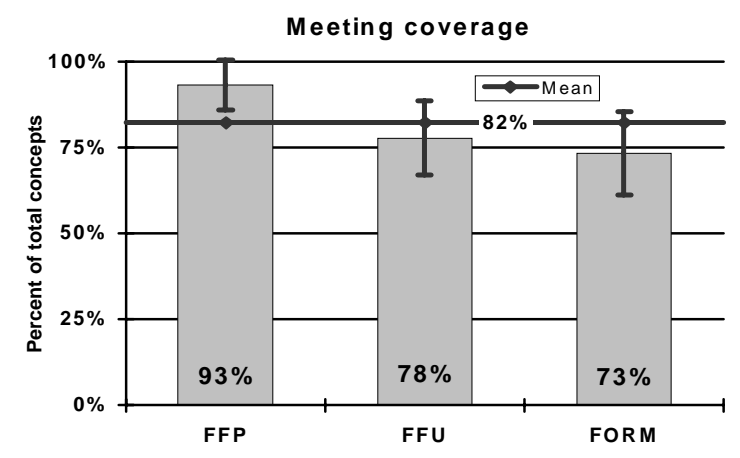

Figure 3. Coverage of meeting concepts in three interface conditions

Multivariate analysis was carried out with the above dependent variables. It was found that the effect of user interface $(\mathrm{F}(10,16)=9.22, \mathrm{p}<0.0001)$ and of experience were statistically significant $(F(5,8)=4.20, p<0.05)$. User interface condition was found to have significant univariate effects on meeting coverage NCOV $(\mathrm{F}(2,12)=5.66, \mathrm{p}<0.05)$ which was higher in free-form than in fixed-form conditions (Figure3).
Experience was found to have significant univariate effects on number of "trust" strategies TRUSTR $(\mathrm{F}(2,12)=5, \mathrm{p}<0.05)$ (Figure 4) and a borderline significant effect on depth of search CATSD $(\mathrm{F}(1,15)=3.21, \mathrm{p}<0.1)$ (Figure 5). Experts used categorization-based strategies more often and took fewer steps to find information when using categories.

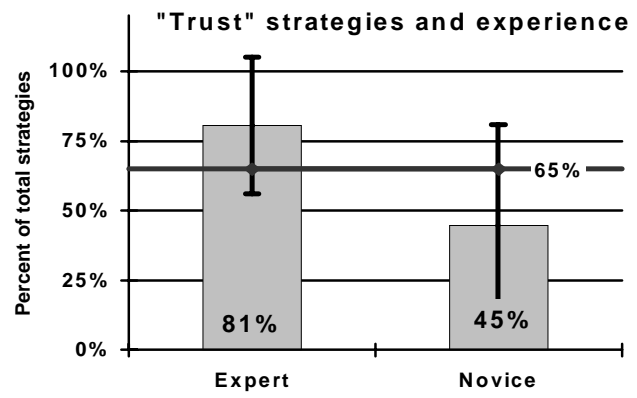

Figure 4. "Trust" strategies in note retrieval by experts and novices

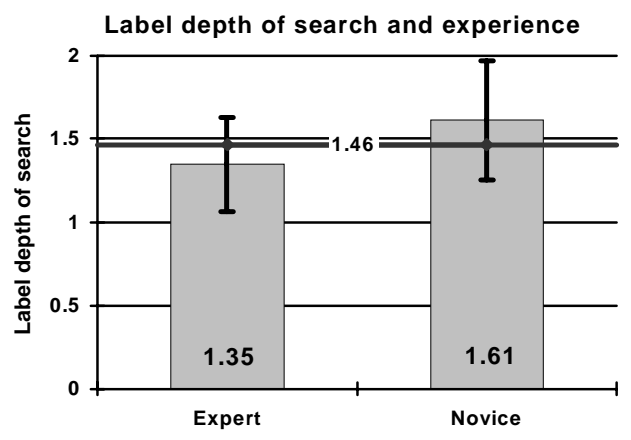

Figure 5. Label depth of search and experience

To gain further insight into the note-taking process qualitative analyses were performed based on observations, interviews with participants, and on content analysis of their notes.

It was observed that forms required thematic note taking, but participants were used to taking notes sequentially. In effect all participants in the FORM condition had problems with selecting forms and appropriate fields within the forms. They wrote notes on unrelated forms that just happened to be open, often using "wrong" fields. Some participants put many notes into one field on a form, others put notes related to the same topic on several forms. Furthermore, forms prevented participants from marking notes and from using drawings.

We also observed that forms forced unnecessary detailed structuring. We found that structuring after note taking (i.e. in free-form interfaces) was easier. However, it did not make information categorization much easier, and difficulties were observed in both free-form and fixed-form interfaces. To deal with categorization problems, participants adopted two strategies: use of a 'miscellaneous' container (the same label applied in 
dubious cases - 5 of 14 participants), and gross categorization (several concepts under one label).

In the information retrieval session categorizations were reapplied. Participants had difficulty in recalling the categories used in their initial classification of content. A couple of participants in the second free-form condition complained about not remembering the meaning of the terms that they themselves had defined.

In addition, we analyzed terminology used by participants for labels in the FFU condition. Participants tended to use concrete terms, both for labels and page titles (Figure 6), while pre-defined terminology was abstract. Furthermore, we examined the reuse of userdefined terms and found that reuse of abstract terms was higher than reuse of concrete terms (Figure 7).

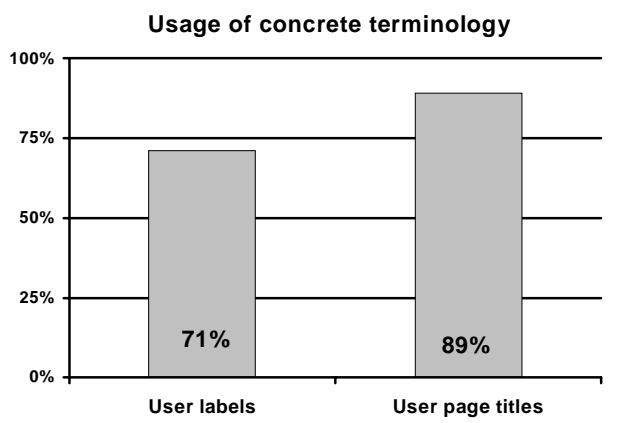

Figure 6. Usage of concrete terminology in the FFU condition

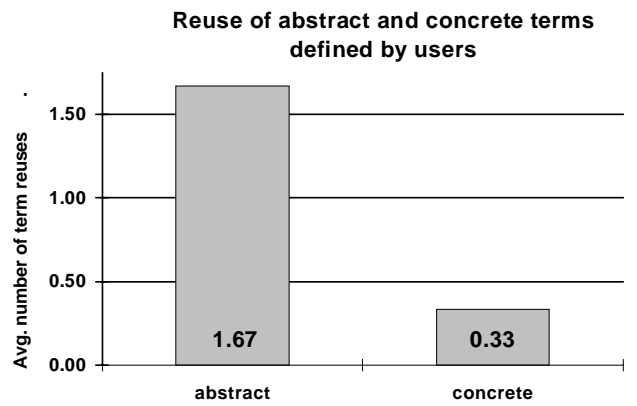

Figure 7. Reuse of user-defined abstract and concrete terms (FFU condition)

\section{DISCUSSION}

Note-taking. The free-form interface appeared to be easier and more natural for note taking, but was not necessarily quicker than fixed-form. The fixed-form interface forced participants to modify their note-taking habits, while the free-form interface did not. As a result notes were more complete in the free-form conditions than in the fixed-form condition (Figure 3).

Structuring Notes. Results from this experiment indicated that, to compare interfaces with regard to information structuring, one needs make a distinction between the information structuring mechanisms, used to separate elements of structure versus the information categorization required by semantic structuring. While the structuring mechanism is a function of the user interface, the terminology used for semantic categorizations is independent of the user interface.

The observations confirmed, that, by virtue of delayed structuring, the free-form interface was easier to use than forms with fixed structure which forced users to break down notes during their creation. Difficulties with categorization were observed in both free-form and fixed-form interfaces.

Participants in those conditions employing domainbased terminology had problems with differentiating and using these predefined terms. These problems were partly explained by the abstractness of the terminology provided, which contrasted with the concrete terms defined by users (Figure 6). Abstract terms were highly reusable (Figure 7), but difficult to understand. On the other hand, concrete terms were easier to understand, but, because of their specificity, not reusable, and thus created a potential difficulty with managing large numbers of terms.

How much categorization is enough? We observed many problems with detailed categorization. Participants were not used to performing detailed categorizations of their notes, since they typically employed other means of accessing notes in their note-taking practice. For example, they used page layout and special marks to visually perceive page structure; they used event memory to locate specific information. The combination of disparate and, at the same time, complementary methods allowed them to effectively find information in the notes. The fixed-form interface forced participants to create more detailed structure than free-form interfaces. Much of that structure was unnecessary, since it did not increase the number of design meeting concepts structured in the FORM condition (Figure 3).

The purpose for providing structure and categories should be considered. Observations from this experiment seem to indicate that facilitating human information processing and facilitating machine information processing through structure require very different approaches. Detailed categories may be good for machine information processing, however, they seem to be very difficult to use by people, and thus not appropriate for facilitating human information retrieval from notes.

The user task also needs to be considered. In the context of taking notes from a design project meeting, a small project (like the one used in this experiment) may require fewer categories than a large one. Participants were overwhelmed with the number of concepts provided for information categorization. 
Expert use of terminology. Experts had less problems with terminology (both with applying it, as well as with using it during information retrieval). Experts used categorization-based search ("trust" strategies) more often then novices (Figure 4), and were able to find information in a smaller number of categorization-based steps (Figure 5).

\section{CONCLUSIONS}

This research was motivated by problems with accessing engineering design information generated in the initial design stages and traditionally recorded in paper engineering notebooks. The focus was on nonintrusive methods of semantic information structuring facilitating subsequent information access.

Experiments were conducted in which free-form and fixed-form note-taking interfaces were compared with respect to capturing and structuring notes from a design meeting. Suitability of domain-based and user-defined terminologies for semantic structuring was also tested.

Based on the analyses of the experiment results we made the following recommendations for the design of electronic engineering notebooks and for the experimental methodologies.

Experimental methodology. Engineering design experience was used as a controlled variable in this study. Our observations indicated that other factors may be more appropriate for consideration in addition to experience. Note-taking habits and organization abilities were idiosyncratic and ideally should be taken into account as well. The difficulty lies in obtaining their objective measure. In future, these could possibly be obtained by observing participants and analyzing the notes taken by them in the course of several note-taking sessions.

Design of Electronic Notebooks. The EEN interface should be extended and made more flexible to accommodate individual differences in note-taking habits. A flexible set of terms should be selected according to user experience and to the type and size of a design project.

The foregoing observations of terminology usage suggest that both terminology types should be combined in one interface; providing pre-defined, domain-based terms, and allowing users to add their own terms.

Abstract terminology should be pre-defined and used for lower level details, while concrete terminology should be user-defined and used for higher level elements in the notebook structure. Employing user-defined terminology for higher level elements would help to limit their number and to make user terminology better manageable.
In the future work, we plan to implement the above recommendations, to perform long term note-taking studies in a professional engineering environment, and to explore effects of common, pre-defined terminologies and user-defined terminologies on sharing design notes.

\section{ACKNOWLEDGEMENTS}

This research was conducted as part of the author's M.A.Sc. research in the Department of Mechanical and Industrial Engineering at the University of Toronto. The author wishes to thank Professor Mark Fox for guidance and support and Professor Mark Chignell for help in the experimental design and for helpful comments on the paper. This research was funded, in part, by PRECARN Associates and Spar Aerospace.

\section{REFERENCES}

Baudin, C., Underwood, J.G., Baya, V. (1993). Using Device Models to Facilitate the Retrieval of Multimedia Design Information. In Proceedings of the 13th International Joint Conference on Artificial Intelligence, (Chambery, France, August 29-September 2,1993) (pp. 1237-1243).

Erickson T. (1996). The design and long-term use of a personal electronic notebook: A reflective analysis", In Proceedings of CHI'96 (pp. 11-18). New York, NY: ACM Press.

Gwizdka, J., Dalal, I. (1996). Action Item Management System, Unpublished Report, Enterprise Integration Laboratory, Department of Industrial Engineering, University of Toronto, Toronto, ON, Canada.

Hong, J., George Toye, G., Leifer, L.J. (1995). Personal Electronic Notebook with Sharing. In Proceedings of the Fourth WET ICE'95 (pp. 88-94). Washington, DC: IEEE Computer Society Press.

Lakin F., Baya, V., Cannon, D.M., Brereton, M., Leifer, L. J., Toye, G. (1992). Mapping Design Information. In Working Notes form the AAAI'92 Workshop on Design Rationale Capture and Use. Menlo Park, CA: AAAI Press.

Lin J., Fox M.S., Bilgic T. (1996). A Requirement Ontology for Engineering Design. In Concurrent Engineering: Research and Applications. 4(3). 279-291.

Louie, J. (1995). An Analysis of a Pen-based Tool for Acquiring Engineering Design Information. (Technical Report TRE-EIL-95-1). Toronto, ON, Canada: Enterprise Intergration Laboratory, Department of Industrial Engineering, University of Toronto.

Olson G.M. Olson, J.S., Storrosten, M., Carter, M., Herbsleb, J., Rueter, H. (1996). The Structure of Activity During Design Meetings. In T.P. Moran and J.M. Carroll (Eds.) Design Rationale: Concepts, Techniques, and Use (pp. 393-427). Hillsdale, NJ: Lawrence Erlbaum Associates.

Wilcox, L., Schilit, B.N., Sawhney, N. (1996). Dynomite: A dynamically organized ink and audio notebook (pp. 186-193). In Proceedings of CHI'96. New York, NY: ACM Press. 